\title{
The harvesting of small molecule structure and dynamic data for macromolecular and catalytic usage.
}

\author{
A. Brink \\ ${ }^{1}$ Chemistry Department, University of the Free State, Bloemfontein, South Africa, 9301 \\ Brinka@ufs.ac.za
}

The various aspects of drug design or catalysis is compartmentalized within defined research fields, i.e. bioactivity testing versus pure synthetic chemistry; homogeneous versus heterogeneous catalysis. These are independent and often non-interactive specialities which have developed along parallel pathways with a common objective. The world economic drive towards the $4^{\text {th }}$ industrial revolution captures the idea of the confluence of new technologies and their cumulative impact on our world. Hence the ability to merge, bridge and remove boundaries will result in the establishment of interoperable research.

Drug design, particularly the development of target specific radiopharmaceuticals which involves the selective receptor binding of a radioactive organometallic complex to a possible disease site involves multiple facets. Simple manipulation of the ligand system bound to the metal centre can significantly alter parameters such as steric and electronic character, chirality, stability, biological and hydro/lipophilicity properties. Our organometallic research utilising the group 7 transition metal triad of manganese, technetium and rhenium for nuclear medical imaging and therapeutic agents, includes the interactions with proteins using protein crystallography. This provides valuable structural information in a similar vein to fragment based drug discovery (FBDD). The domain of chemical versus macromolecular crystallography has resulted in multiple discipline variations, such as incompatible software, data formatting and terminology. A key challenge which hinders research advancement is the lack of interoperability between chemical and biological crystallographic data.

This perspective will highlight the opportunities of harvesting both small molecule and macromolecular structural data, the joint usage of the CSD and PDB databases, as well as the advantages of software which can convert organometallic small molecule structural data for use in protein refinement software. This multidiscipline approach to radiopharmaceutical development will include kinetic reactivity studies highlighting how subtle structural changes can significantly affect chemical reactivity and hence the protein coordination in macromolecular structures. Trends similarly witnessed in catalysis research.

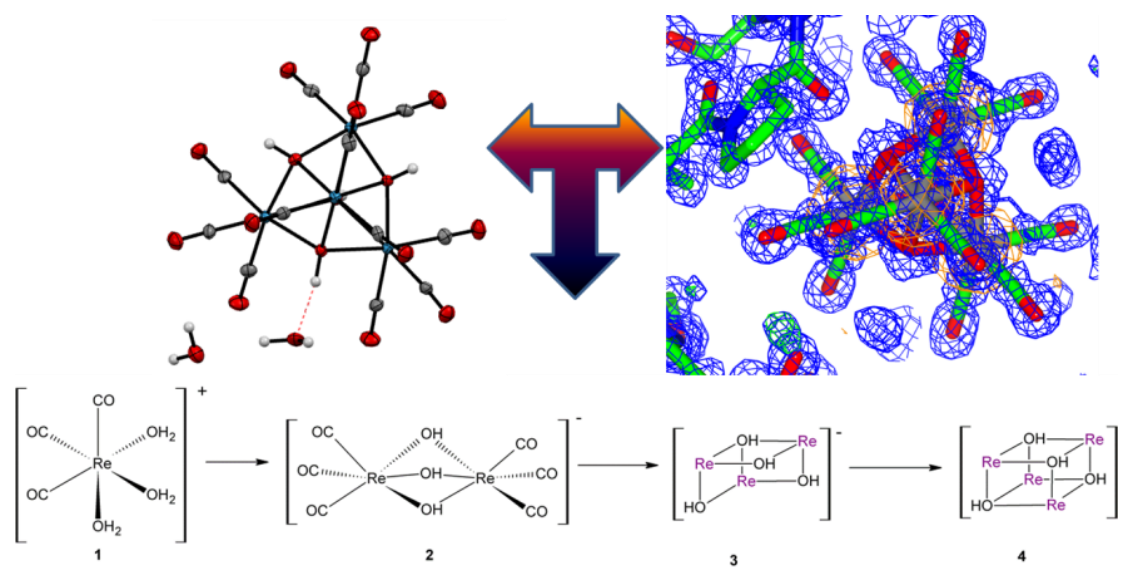

Figure 1. The structure and reaction kinetic usage of chemical and macromolecular data during the formation of tetranuclear rhenium clusters.

[1-3] Brink, A., Helliwell, J.R. (2019). IUCrJ., 6, 788-793. Brink, A., Helliwell, J.R. (2019). IUCrJ., 6, 695-702; Brink, A., Helliwell, J.R. (2017). IUCrJ., 4, 283-290

[4] Alexander, O., Duvenhage, M.M., Kroon , R.E., Brink, A., Visser, H.G. (2021). New J. Chem., 45, 2132-2140

[5] Alberto, R., Frei, A., Mokolokolo, P.P., Bolliger, R., Braband, H., Mampotso, T.S., Brink, A., Roodt, A., (2018). Chemistry: A European Journal, 24, 10397-10402

[6] Jacobs, F.J.F., Venter, G.J.S., Brink, A. (2021). Dalton Trans., unpublished results.

Keywords: Interoperability; radiopharmaceutical drug development; catalysis; rhenium cluster formation 